\title{
IMPORTANCE OF CALIBRATION/VALIDATION TRACEABILITY FOR MULTI-SENSOR IMAGING SPECTROMETRY APPLICATIONS
}

\author{
K. Thome \\ National Aeronautics and Space Administration (NASA) Goddard Space Flight Center, USA
}

\begin{abstract}
Knowledge of calibration traceability is essential for ensuring the quality of data products relying on multiple sensors and especially true for imaging spectrometers. The current work discusses the expected impact that imaging spectrometers have in ensuring radiometric traceability for both multispectral and hyperspectral products. The Climate Absolute Radiance and Refractivity Observatory Pathfinder mission is used to show the role that high-accuracy imaging spectrometers can play in understanding test sites used for vicarious calibration of sensors. The associated Solar, Lunar for Absolute Reflectance Imaging Spectroradiometer calibration demonstration system is used to illustrate recent advances in laboratory radiometric calibration approaches that will allow both the use of imaging spectrometers as calibration standards as well as to ensure the consistency of the multiple imaging spectrometers expected to be on orbit in the next decade.
\end{abstract}

Index Terms - Calibration, validation, imaging spectroscopy, hyperspectral, traceability

\section{INTRODUCTION}

An important aspect to using imaging spectrometer data, and all remote sensing data for that matter, is the characterization and calibration of the sensors and validation of their data products. Just as important is the development of an error budget with known traceability. The radiometric accuracy of a given sensor is important for demonstrating the expected quality from the sensor, but it is its traceability that allows data from multiple sensors to be directly comparable without the need for on-orbit inter-comparisons.

The expected launches of multiple imaging spectrometers from multiple countries, agencies, and commercial entities make knowledge of radiometric traceability an even more important factor. The Climate Absolute Radiance and Refractivity Observatory (CLARREO) combines both the high accuracy needed for climate studies as well as the traceability to allow multiple CLARREO platforms to agree with each other without the need for overlapping data [1]. In 2016, the CLARREO project received funding for a CLARREO Pathfinder (CPF) mission to demonstrate essential measurement technologies required for the full mission through a launch of the reflected solar imaging spectrometer to the International Space Station (ISS) in 2020.

The goal of CPF is to use its planned one year of operation to demonstrate climate-level accuracies, sensor-tosensor inter-calibration, and CLARREO-like retrievals within the latitudinal coverage allowed by the ISS platform. CPF will also impact other missions through developing innovative on-orbit SI-traceable methods and absolute calibration of the lunar spectral irradiance. The final area that CPF will impact will be by demonstrating improved laboratory SI-traceable calibration approaches.

The current work describes how the CPF imaging spectrometer can characterize the ground sites that are currently used for sensor inter-calibration as a demonstration of imaging spectrometers playing a key role in the radiometric calibration of on-orbit sensors. Coupled with the test site characterization is the need to demonstrate SItraceability and the work here gives the example of the laboratory-based calibration demonstration system (CDS) for CLARREO. Both examples are used to show the importance of imaging spectrometry in the future of sensorto-sensor traceability.

\section{TRACEABILITY}

Traceability refers to adherence to an agreed upon standard. There is specific guidance on how traceability should be achieved (Guidelines for Evaluating and Expressing the Uncertainty of NIST Measurement Results [2]). The most basic view of traceability is that an error budget determines the uncertainty relative to the agreed-upon standard. SItraceability is achieved if that standard is part of the Systeme International. The advantage of developing a truly SItraceable error budget is that the same physical measurement from different sensors will be directly comparable.

The complexity of sensors and scenes in earth sciences, coupled with possible degradation during the mission, makes traceability more important to allow direct comparisons of data products. A common radiometric scale is the key to ensuring the consistency of as many sensors as possible, including the expected range of imaging spectrometers expected to be launched in the near future. Obtaining 
consistency through SI-traceable approaches leads to comprehensive sets of long-term, consistent, and calibrated data and products. Developing traceable methods allows data from sensors that do not have temporal overlap to be combined. Such traceability efforts must make use of hyperspectral data sets to limit uncertainties caused by knowledge of the spectral response of the many sensors to be calibrated.

An SI-traceable approach with emphasis on accuracy determination following the lessons learned from the national metrological institutes would lead to the needed sensor agreement. The effort can rely on an on-orbit sensor with known absolute uncertainty and defensible traceability to act as a transfer standard. Equivalently, well established methods for prelaunch calibration with appropriate uncertainties coupled with SI-traceability would lead to agreement between sensors once they are on orbit and assuming no changes in going to orbit. The last approach is to use highly accurate sensors to characterize test sites that can then be used as transfer standards.

\section{TEST SITE CHARACTERIZATION}

The inter-calibration efforts of CPF will concentrate on a limited number of sensors due to budget and mission lifetime constraints. The goal will be to demonstrate that CLARREO-level accuracies can be transferred in an SItraceable fashion to other sensors. CPF and CLARREO inter-calibration is unique in that the sensor will be pointed to match both the areal coverage and the sun-sensor geometry of the sensor to be characterized. The imaging spectrometer nature of CPF allows spectral convolution of the CPF data to match the spectral response of the other sensor. The spectral resolution and sampling of CPF has been selected to allow a full range of scene types including clouds, clear-sky ocean, snow/ice, and vegetation to be used along with those desert scenes typically thought of for radiometric inter-calibration.

The CLARREO approach to intercalibration mitigates the issues caused by changing illumination and view conditions as well as physical changes in the atmosphere and surface over time. Even a sensor specifically tasked with only the inter-calibration would not be able to image match all sensors. An alternative approach, similar to that proposed by Teillet et al. [3], is to have a system like CPF calibrate a test site that is then used to calibrate the sensor of interest. The method depends upon the use of imaging spectrometry to account for both the spatial and spectral nature of the test site [3].

Such a site calibration approach has been used to intercompare data from a variety of sensors viewing a test site at various times on the same date with varying view angles [4]. Past work has shown that site characterization using groundbased sensors allows inter-calibration of sensors without coincident views and geometry [5]. A key to the above methods and recent methods of cross-comparisons is the use of hyperspectral data sets to allow convolution of the atsensor radiance across the specific spectral bands of a given sensor.

More recent activities have led to greater understanding of the behavior of test sites as a function of time including solar and view angle geometry, spectral stability, and atmospheric behavior. Coordination of multiple sensors has allowed hyperspectral measurements to be made of key test sites. Multi-angle data sets permit greater understanding of surface directional reflectance effects. Monitoring by Aeronet radiometers is helping to parameterize atmospheric conditions.

Inclusion of data from future sensors such as CLARREO, CPF, and TRUTHS (Traceable Radiometry Underpinning Terrestrial- and Helio- Studies) [6] are necessary to permit enhanced understanding of the test sites. The proposed requirements for such sensors are absolute uncertainties $<0.3 \%$ in band-integrated albedo to allow those sensors to be used in the development of climate benchmark data sets. The high accuracy and spectral sampling of such sensors is vital to allow separation of surface effects from atmospheric effects permitting the development of the needed models for test site calibration. Similarly well-calibrated and characterized ground-based instrumentation and airborne sensors are likewise needed to improve site assessments.

The absolute uncertainty of such a site calibration method would not supply results of the same accuracy as CPF. However, it should be recognized that not all sensors require the full accuracy of CLARREO or CPF. The site calibration approach can rely on CPF to characterize those test sites already being used as pseudo-invariant test sites such as the Libyan Desert [7]. Further, CPF can be used to characterize instrumented test sites such as those in France, China, and the United States to give a clearer understanding of the uncertainties caused by atmospheric and surface bidirectional effects [8].

\section{PRELAUNCH RADIOMETRIC CALIBRATION}

The above cannot occur without a well characterized and calibrated sensor prior to launch. CPF will demonstrate improved laboratory SI-traceable calibration approaches. The approaches being developed for CPF can have applicability to other missions as evidenced by the use of these methods on a smaller scale in recent sensor characterizations [9]. The SOlar, Lunar for Absolute Reflectance Imaging Spectroradiometer (SOLARIS) CDS for CLARREO is being used as a test bed for these approaches and will play a role in demonstrating the SItraceability of CPF [10].

The calibration approach for the CLARREO mission relies on a direct solar view as the primary calibration approach while on orbit. The data from a solar view are coupled with the earth view data and knowledge of the 
sensor optical geometry to retrieve at-sensor reflectance. SI traceability is the cornerstone of the success of CLARREO's mission. The basis for the long-term SI-traceability on orbit is the sun itself through the determination of reflectance. The demonstration of SI traceability requires both a detailed preflight calibration and then a transfer of that calibration to orbit. It is expected that the primary sources of error in transferring the prelaunch calibration to orbit will be changes in stray light behavior and polarization sensitivity.

Prelaunch laboratory measurements are critical to the prediction of the on-orbit sensor output via a sensor model. Disparities between laboratory results and model predictions guide model improvements. A critical part of the calibration process is development of an SI-traceable data set that is achieved by characterizing the sensor to SI-traceable, absolute radiometric quantities during prelaunch calibration, primarily the electric Watt. Prelaunch absolute calibration includes both irradiance and radiance modes as well as the determination of geometric factors for conversion to reflectance.

Calibration systems, such NIST's Spectral Irradiance and Radiance Responsivity Calibrations using Uniform Sources (SIRCUS) facility, provide such standards and a capability to understand stray light, spectral response, and polarization sensitivity at the level necessary for CLARREO [11]. The basis of SIRCUS is a well-understood tunable laser source that can be coupled to a fiber optic system providing both radiance and irradiance sources. The output of the source is determined via detector standards characterized against the Primary Optical Watt Radiometer (POWR). The calibration traceability to SIRCUS begins with a substitution radiometer that is used to calibrate the tunable laser source, known as the POWR Laser. In a second step, the POWR unit is moved and replaced by the CLARREO Transfer Radiometer (CXR) based on a silicon-trap detector for the visible and near infrared and indium-gallium arsenide detectors at longer wavelengths. The stated accuracy to calibrate a transfer radiometer in irradiance mode using POWR is $0.09 \%$ at $\mathrm{k}=3$.

Applying this detector-based approach to the laboratory calibration of SOLARIS leads to an end-to-end instrument performance model and error budgets with measured uncertainty magnitudes and peer reviewed measurement accuracy traceability chains all of which are applicable to CLARREO. SOLARIS gives the opportunity to include NIST-based laboratory calibrations both with monochromatic (SIRCUS) and broadband calibration approaches. Key accomplishments with SOLARIS to date include:

1) Absolute spectral response obtained via SIRCUS

2) Evaluation of polarization measurement approaches

3) Component-level characterization of optical elements

4) Field-based radiance measurements of the sun, moon, and desert sites
5) NIST-evaluation of the SOLARIS test plan and early SOLARIS laboratory results

A physically-based spectrometer model will demonstrate the capability to characterize SOLARIS and predict its behavior giving confidence to do the same with CPF both prelaunch and on orbit. Ultimately, SOLARIS results will, as the name Calibration Demonstration System implies, lead to a demonstration that CLARREO-level accuracies are achievable and defensible. Further, SOLARIS testing is designed to achieve "operational" use of SIRCUS-type calibration approaches and this will provide the basis for other groups to implement similar methods in their own facilities.

\section{CONCLUSIONS}

The combination of high accuracy, SI-traceability, and imaging spectrometry offers opportunities to understand a variety of on-orbit sensors using inter-calibration approaches and improvements to prelaunch calibration. Inter-calibration methods have improved dramatically in recent years because of the use of imaging spectrometry. The inclusion of highaccuracy reference standards such as CLARREO, CPF, and TRUTHS will improve both the traceability and the accuracy of direct inter-calibrations with on-orbit sensors. The imaging spectrometer nature of these reference standards is a critical aspect to providing a sufficient number of data sets for suitable inter-calibration results.

The imaging spectrometer reference standards also make it possible to consider placing ground test sites on an SI-traceable scale that allows their use at a level of precision and accuracy once only considered possible for lunar measurements. Coordinated international efforts to understand the ground sites will permit a physically-based understanding allowing predictions of at-sensor radiance with absolute uncertainties $<1 \%$.

The hope is that demonstrating the utility of highly accurate, SI-traceable sensors will convince others of the importance to follow suit. Demonstrating the prelaunch methods being proposed for CPF and making the methods operational will allow the approaches to be implemented in multiple facilities. The results from the testing of SOLARIS in the laboratory will be the first step to developing techniques as straightforward as the current lamp-based calibration techniques.

\section{REFERENCES}

[1] B.A. Wielicki, D. F. Young, M. G. Mlynczak, K. J. Thome, S. Leroy, J. Corliss, J. G. Anderson, C. O. Ao, C.O., R. Bantges, F. Best, K. Bowman, H. Brindley, J. J. Butler, W. Collins, J. A. Dykema, D. R. Doelling, D. R., Feldman, N. Fox, X. Huang, R. Holz, Y. Huang, Z. Jin, D. Jennings, D. G. Johnson, K. Jucks, S. Kato, D. B. Kirk-Davidoff, R. Knuteson, G. Kopp, D. P. Kratz, X. 
Liu, C. Lukashin, A. J. Mannucci, N. Phojanamongkolkij, P. Pilewskie, V. Ramaswamy, H. Revercomb, J. Rice, Y. Roberts, C. M. Roithmayr, F. Rose, S. Sandford, E. L. Shirley, W. L. Smith Sr., B. Soden, P. W. Speth, W. Sun, P. C. Taylor, D. Tobin, and X. Xiong, "Achieving Climate Change Absolute Accuracy in Orbit," Bulletin of the American Meteorological Society 94, 1519-1539 2013.

[2] B. N. Taylor, C. E. Kuyatt, "Guidelines for Evaluating and Expressing the Uncertainty of NIST Measurement Results." NIST TN 1297, 20 pp., 1994.

[3] P. M. Teillet, G. Fedosejevs, R. P. Gauthier, N. T. O’Neill, K. J. Thome, S. F. Biggar, H. Ripley, and A. Meygret, "A generalized approach to the vicarious calibration of multiple Earth observation sensors using hyperspectral data," Remote Sensing of Environment, 77, pp. 304-327, 2001.

[4] K. J. Thome, S. F. Biggar, W. T. Wisniewski, "Crosscomparison of EO-1 sensors and other Earth Resources Sensors to Landsat-7 ETM+ Using Railroad Valley Playa," IEEE Trans. On Geosciences and Remote Sensing, Vol. 41, pp. 1180-1188, 2003.

[5] K. J. Thome, J. McCorkel, and J. Czapla-Myers. 2013. "In-Situ Transfer Standard and Coincident-View Intercomparisons for Sensor Cross-Calibration." IEEE Transactions on Geoscience and Remote Sensing, 51, pp. 1088-1097 2013.

[6] Fox, N, A. Kaisser-Weiss, W. Schmutz, K. Thome, D. Young, B. Wielicki, R. Winkler, E. Woolliams, 2011: Accurate Radiometry from Space: an essential tool for climate studies, Phil. Trans. R. Soc. A, 369, 4028-4063 doi:10.1098/rsta.2011.0246

[7] J. McCorkel, K. J. Thome, and R. B. Lockwood. "Absolute Radiometric Calibration of Narrow-Swath Imaging Sensors With Reference to Non-Coincident Wide-Swath Sensors." IEEE Trans. Geosci. Remote Sensing, 51, pp. 1309-1318, 2013.

[8] J. Czapla-Myers, K. J. Thome, and N. P. Leisso. "Radiometric calibration of earth-observing sensors using an automated test site at Railroad Valley, Nevada." Canadian Journal of Remote Sensing, 36, pp. 474-487, 2010.

[9] H. Oudrari, J. McIntire, X. Xiong, J. Butler, S. Lee, N. Lei, T. Schwarting, and J. Sun, "Prelaunch radiometric characterization and calibration of the S-NPP VIIRS sensor," IEEE T. Geosci. Remote Sens., 53, pp. 2195, 2015.

[10] K. Thome, McCorkel, J., Hair, J., McAndrew, B., Daw, A., Jennings, D., and Rabin, D. "Test plan for a calibration demonstration system for the reflected solar instrument for the climate absolute radiance and refractivity observatory," Proc. SPIE 8516, 1-11, 2012.

[11] Brown, S.W., Eppeldauer, G.P., and Lykke, K.R., "NIST facility for Spectral Irradiance and Radiance Responsivity Calibrations with Uniform Sources," Metrologia Vol. 37, 579-582, 2000. 